\title{
Protocol
}

\section{Microinjection of dsRNA in Tardigrades}

\author{
Jennifer R. Tenlen ${ }^{1}$ \\ Department of Biology, Seattle Pacific University, Seattle, Washington 98119
}

\begin{abstract}
Classical genetic analysis in the tardigrade Hypsibius exemplaris is a challenge because these animals are parthenogens. The publication of the H. exemplaris genome has facilitated the study of targeted genes by RNA interference (RNAi), a robust mechanism to disrupt gene function. This protocol describes microinjection of double-stranded RNA (dsRNA) in tardigrades using techniques adapted from protocols originally developed in Caenorhabditis elegans. A DNA template (either genomic or cDNA) is used to prepare dsRNA, to which T7 polymerase binding sites are added at the $5^{\prime}$ end of each strand. The dsRNA is injected into adult tardigrades, preferably targeting the gonad or intestine. Injected adults are allowed to recover in spring water and then transferred to culture dishes or individual wells of a 96-well plate.
\end{abstract}

It is essential that you consult the appropriate Material Safety Data Sheets and your institution's Environmental Health and Safety Office for proper handling of equipment and hazardous materials used in this protocol.

Algae (Clorococcum sp.) (Sciento)

Ethanol (95\%)

Ethanol (70\%, ice-cold)

Genomic DNA or cDNA containing the region of interest

$\mathrm{H}_{2} \mathrm{O}$ (nuclease-free)

Halocarbon 700 oil (Halocarbon Products)

In vitro transcription kit

Levamisole (tetramisole hydrochloride; Sigma-Aldrich L9756) (50 mm in $\mathrm{H}_{2} \mathrm{O}$ )

Use of levamisole as an anesthetic is optional.

Reagents and primers for PCR

For amplification of the region of interest and addition of $T 7$ polymerase binding sites (Step 1), polymerase chain reaction (PCR) primers should start with the T7 sequence [5'-CATAATACGACTCACTATAGGG-3'], followed by 15-18 nucleotides of gene-specific sequence.

Sodium acetate (3 $\mathrm{m}, \mathrm{pH} 5.2)$

Spring water

Tardigrades (adults, in spring water)

For general advice on maintaining tardigrades in the laboratory, see Protocol: Laboratory Culture of Hypsibius exemplaris (MCNuff 2018).

\footnotetext{
${ }^{1}$ Correspondence: tenlenj@spu.edu

From the Emerging Model Organisms collection.

(c) 2018 Cold Spring Harbor Laboratory Press

Cite this protocol as Cold Spring Harb Protoc; doi:10.1101/pdb.prot102368
} 
Equipment

Capillaries (borosilicate glass, 1.0-mm OD, 0.58-mm ID, inner filament) (World Precision Instruments 1B100F-4)

The inner filament promotes backfilling by capillary action.

Clay (modeling)

Glass cutter

Glass depression slide (two-well)

Inverted microscope for microinjections

Microcentrifuge

Microcentrifuge tubes

Micromanipulator

Micropipette puller

Microscope cover glass $(22 \mathrm{~mm} \times 22 \mathrm{~mm}$, No. 11/2)

Microscope slides (glass)

Nail polish (clear)

Petri dish (100-mm)

Spectrophotometer

Stereomicroscope

Tissue culture plates (96-well, round bottom, untreated, sterile) (Greiner Bio-One 650-185) or 35mm Petri dishes (see Step 23)

\section{METHOD}

Preparation of dsRNA

1. Amplify the region of interest from genomic DNA or CDNA in a PCR that attaches T7 polymerase binding sites to the $5^{\prime}$ end of each strand.

We have had success with PCR products in the range of 275-1000 bp.

2. Use $1 \mu \mathrm{g}$ of the final DNA product from Step 1 as the template to synthesize dsRNA via an in vitro transcription kit, following the manufacturer's directions.

3. To purify the dsRNA, add 0.1 volume of $3 \mathrm{~m}$ sodium acetate and 2.5 volumes of $95 \%$ ethanol to the sample from Step 2. Incubate on ice for $5 \mathrm{~min}$ and then centrifuge at maximum speed in a microcentrifuge for $10 \mathrm{~min}$.

4. Remove the supernatant and then wash the pellet with $0.5 \mathrm{~mL}$ of ice-cold $70 \%$ ethanol. Centrifuge at maximum speed in a microcentrifuge for $5 \mathrm{~min}$. Remove the supernatant.

5. Air-dry the pellet at room temperature, and then resuspend the dsRNA in nuclease-free $\mathrm{H}_{2} \mathrm{O}$.

6. Determine the concentration of dsRNA via spectrometry.

The extinction coefficient for dsRNA is 45 .

7. Store the dsRNA solution at $-70^{\circ} \mathrm{C}$.

Preparation of Needles for Microinjection

8. Use a micropipette puller to pull needles to a fine taper from borosilicate glass tubing (1.0-mm $\mathrm{OD}, 0.58-\mathrm{mm} \mathrm{OD})$. Follow the manufacturer's instructions to obtain needles that taper quickly into a sharp point, with a bore of about $1 \mu \mathrm{m}$.

To achieve a good needle, it may be necessary to adjust the pulling force and/or the temperature of the heating filament. If the temperature and/or the pulling force are too low, then the needle may have a long taper that is too flaccid to penetrate the tardigrade's cuticle. Conversely, if either parameter is too high, then the needles could result in a bore size that is too large for injections. 
J.R. Tenlen

9. Store the needles on a long piece of clay molded into the bottom of a 100-mm Petri dish and keep covered until needed.

10. For microinjection experiments, dilute the dsRNA to a final concentration of $1 \mu \mathrm{g} / \mu \mathrm{L}$ in nucleasefree $\mathrm{H}_{2} \mathrm{O}$. Centrifuge briefly to clear the supernatant before filling the injection needle.

11. Back-fill the injection needle with dsRNA, and allow the dsRNA to flow to the tip of the needle by gravity.

\section{Microinjection of Animals}

12. Prepare the injection chamber as follows.

i. Use a glass cutter to remove the corner of a microscope cover glass $(22 \mathrm{~mm} \times 22 \mathrm{~mm}$, No. $\left.1 \frac{1}{2}\right)$.

ii. Place the triangular corner piece in the middle of a standard glass microscope slide, such that one straight edge is parallel to the short edge of the slide (Fig. 1).

iii. Secure the cover glass to the microscope slide with clear nail polish.

The purpose of the cover glass is to create a solid barrier against which to brace the animals. Imperfections in the cover glass can sometimes create a gap between the cover glass and the microscope slide large enough for the animals to slip under. Mounting corners of cover glass, rather than a whole piece, appears to result in fewer gaps.

Chambers can be prepared in advance and stored in a microscope slide box.

13. Using a stereomicroscope, transfer adult tardigrades into one well of a deep two-well depression slide. Allow animals to excrete any material from their intestines, changing the spring water several times.

This step can take up to $30 \mathrm{~min}$.

14. (Optional) Anesthetize the animals in levamisole immediately before performing injections by replacing the spring water with $10 \mathrm{~mm}$ levamisole (prepared in spring water).

This step helps to minimize animal movement during microinjections (Tenlen et al. 2013) but is not required (Boothby et al. 2017).

15. Apply a drop of Halocarbon 700 oil to the straight edge of the cover glass on the injection chamber.

16. Using a stereomicroscope, transfer several animals to the oil drop on the prepared injection chamber. Position each animal lengthwise along the straight edge of the cover glass. Remove any residual water.

We typically inject 5-10 animals at a time; animals appear to be unaffected by exposure to halocarbon oil.

17. Mount the microinjection needle in the needle holder of the micromanipulator.

18. Position the injection chamber on the inverted microscope and orient the micromanipulator so that the needle is perpendicular to the tardigrade (Fig. 1). Under the lowest-power objective, bring both the animal and needle in focus.

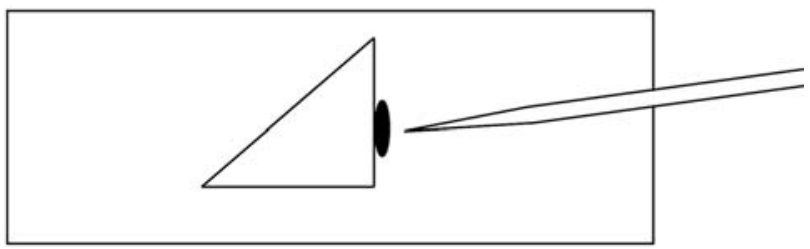

FIGURE 1. Schematic of injection chamber. 
19. Switch to the $20 \times$ objective and lower the needle into position. To break the needle, use the micromanipulator controls to bring the needle into the same focal plane as the cover glass edge, then gently brush (stroke) the needle against the coverslip edge.

20. Using the micromanipulator fine controls, adjust the needle so that it is in the same focal plane as the animal. Bring the needle in contact with midpoint of the body (so that it is targeting the gonad/intestine) and then gently tap on the left side of the microscope stage to force the needle into the body.

Depending on the bore size, one or two taps of the foot pedal should be sufficient. Delivery of dsRNA can be confirmed by slight swelling at the site of injection.

21. Use the micromanipulator controls to withdraw the needle, and move to the next animal.

Recovery and Monitoring of Animals

22. After all animals on a slide have been injected, move the slide back to the stereomicroscope. Add a drop of spring water over each animal, and then transfer the animals to the second well of the depression slide.

23. To monitor individual animals and their progeny, prepare a 96-well tissue culture plate (untreated, sterile) by adding $100 \mu \mathrm{L}$ of spring water and $5 \mu \mathrm{L}$ of green algae to each well. Transfer one animal to each well.

Animals may instead be transferred in groups to 35- $\mathrm{mm}$ Petri dishes, with a layer of spring water and one to two drops of green algae.

24. Maintain all injected animals at room temperature, and monitor water and food levels regularly.

Parameters established for microinjection in C. elegans, including needle conditions, appear to also be effective in tardigrades (Fire 1986; Fire et al. 1998). Injection of dsRNA into the tardigrade gonad, intestine or coelomic cavity results in loss-of-function phenotypes observed in progeny (Tenlen et al. 2013). This ability of RNAi to cross tissue boundaries was initially observed in C. elegans (Fire et al. 1998). Either genomic DNA or cDNA can be used as templates to synthesize dsRNA. However, the choice of DNA template may need to be empirically determined for each experiment. For some genes, such the $H$. exemplaris actin homolog, similar results were obtained using dsRNA transcribed from either genomic DNA or cDNA templates. Conversely, for other genes, embryonic phenotypes were observed only for injection of dsRNA from cDNA templates.

The recovery and reproductive success of tardigrades following injection appears to be dependent on multiple environmental conditions (such as oxygen content of water, humidity and amount/ quality of algae food source). For each experiment, several controls should be set up at the same time. Negative controls include both uninjected animals and animals injected with $\mathrm{H}_{2} \mathrm{O}$ or dsRNA targeting green fluorescent protein (Tenlen et al. 2013). Hd-actin is recommended as a positive control since injection of $H d$-act dsRNA results in highly penetrant lethality in progeny, with reproducible cytokinesis defects (Tenlen et al. 2013).

In our experiments, the penetrance of loss-of-function phenotypes varies based on the targeted gene, and on brood number. Reproduction and molting are linked in tardigrades, occurring approximately every $4-5 \mathrm{~d}$. When injection of dsRNA resulted in a phenotype, the penetrance was always highest in the first brood of embryos produced after injection. In subsequent broods, the penetrance decreased substantially (Tenlen et al. 2013). As a result, it may be necessary to increase the number of adults injected to ensure an adequate population of embryos to analyze. 


\section{ACKNOWLEDGMENTS}

J.R.T. was supported by grants from the National Institutes of Health (K12GM000678), the NIHfunded UNC Developmental Biology Training Program (T32HD046369-03), and the M. J. Murdock Charitable Trust.

\section{REFERENCES}

Boothby TC, Tapia H, Brozena AH, Piszkiewicz S, Smith AE, Giovannini I, Rebecchi L, Pielak GJ, Koshland D, Goldstein B. 2017. Tardigrades use intrinsically disordered proteins to survive desiccation. Mol Cell 65: 975-984.

Fire A. 1986. Integrative transformation of Caenorhabditis elegans. EMBO J 5: $2673-2680$.

Fire A, Xu SQ, Montgomery MK, Kostas SA, Driver SE, Mello CC. 1998. Potent and specific genetic interference by double-stranded RNA in Caenorhabditis elegans. Nature 391: 806-811.
McNuff R. 2018. Laboratory culture of Hypsibius exemplaris. Cold Spring Harb Protoc doi:10.1101/pdb.prot102319.

Tenlen JR, McCaskill S, Goldstein B. 2013. RNA interference can be used to disrupt gene function in tardigrades. Dev Genes Evol 223: 171-181. 


\section{Microinjection of dsRNA in Tardigrades}

Jennifer R. Tenlen

Cold Spring Harb Protoc; doi: 10.1101/pdb.prot102368

Email Alerting Receive free email alerts when new articles cite this article - click here.

Service

Subject Browse articles on similar topics from Cold Spring Harbor Protocols.

Categories

Developmental Biology (728 articles)

Emerging Model Organisms (321 articles)

Molecular Biology, general (1293 articles)

RNA (317 articles)

RNA Interference (RNAi)/siRNA (123 articles)

RNA, general (269 articles) 